\title{
Article \\ Effects of Epstein-Barr Virus Infection on the Risk and Prognosis of Primary Laryngeal Squamous Cell Carcinoma: A Hospital-Based Case-Control Study in Taiwan
}

\author{
Li-Ang Lee ${ }^{1,2,+}+{ }^{\circ}$, Tuan-Jen Fang ${ }^{1,2,+}{ }^{+}$, Hsueh-Yu Li ${ }^{1,2}$, Hai-Hua Chuang ${ }^{2,3,4}$, Chung-Jan Kang ${ }^{1,2}$, \\ Kai-Ping Chang ${ }^{1,2}$, Chun-Ta Liao ${ }^{1,2}$, Tse-Ching Chen ${ }^{2,5}$, Chung-Guei Huang ${ }^{6,7, *}$ and Tzu-Chen Yen ${ }^{2,8, *}$
}

1 Department of Otorhinolaryngology_-Head and Neck Surgery, Chang Gung Memorial Hospital, Linkou Main Branch, Taoyuan City 33305, Taiwan; 5738@cgmh.org.tw (L.-A.L.); fang3109@cgmh.org.tw (T.-J.F.); hyli38@cgmh.org.tw (H.-Y.L.); keny@cgmh.org.tw (C.-J.K.); changkp@cgmh.org.tw (K.-P.C.); liaoct@cgmh.org.tw (C.-T.L.)

2 Faculty of Medicine, Chang Gung University, Taoyuan City 33302, Taiwan; chhaihua@cgmh.org.tw (H.-H.C.); ctc323@cgmh.org.tw (T.-C.C.)

3 Department of Family Medicine, Chang Gung Memorial Hospital, Linkou Main Branch, Taoyuan 33305, Taiwan

4 Department of Industrial Engineering and Management, National Taipei University of Technology, Taipei 10608, Taiwan

check for updates

Citation: Lee, L.-A.; Fang, T.-J.; Li, H.-Y.; Chuang, H.-H.; Kang, C.-J.; Chang, K.-P.; Liao, C.-T.; Chen, T.-C.; Huang, C.-G.; Yen, T.-C. Effects of Epstein-Barr Virus Infection on the Risk and Prognosis of Primary

Laryngeal Squamous Cell Carcinoma: A Hospital-Based Case-Control Study in Taiwan. Cancers 2021, 13, 1741. https://doi.org/10.3390/ cancers13071741

Academic Editors: Lorenzo Leoncini and Lucia Mundo

Received: 16 March 2021

Accepted: 1 April 2021

Published: 6 April 2021

Publisher's Note: MDPI stays neutral with regard to jurisdictional claims in published maps and institutional affiliations.

Copyright: () 2021 by the authors. Licensee MDPI, Basel, Switzerland. This article is an open access article distributed under the terms and conditions of the Creative Commons Attribution (CC BY) license (https:// creativecommons.org/licenses/by/ $4.0 /)$.
5 Department of Pathology, Chang Gung Memorial Hospital, Linkou Main Branch, Taoyuan City 33305, Taiwan

6 Department of Laboratory Medicine, Chang Gung Memorial Hospital, Linkou Main Branch, Taoyuan City 33305, Taiwan

7 Research Center for Emerging Viral Infections, Graduate Institute of Biomedical Sciences, Chang Gung University, Taoyuan City 33302, Taiwan

8 Department of Nuclear Medicine and Molecular Imaging Center, Chang Gung Memorial Hospital, Linkou Main Branch, Taoyuan City 33305, Taiwan

* Correspondence: joyce@cgmh.org.tw (C.-G.H.); yen1110@cgmh.org.tw (T.-C.Y.); Tel.: +886-3328-1200 (ext. 5653) (C.-G.H.); +886-3328-1200 (ext. 2673) (T.-C.Y.)

+ These authors contributed equally to this work.

Simple Summary: Epstein-Barr virus DNA positivity, age $\geq 55$ years, cigarette smoking, and high $B C L-2, B 2 M$, and $C D 161$ expression were identified as independent risk factors for primary laryngeal squamous cell carcinoma. A high EBER signal and low CD3 expression significantly and independently predicted local recurrence and disease-free survival within five years. The information obtained in this study improves our understanding of viral infections in laryngeal cancer, and may guide future prevention, treatment, and follow-up strategies.

Abstract: Mounting molecular evidence supports Epstein-Barr virus (EBV) involvement in the pathogenesis of laryngeal squamous cell carcinoma (LSCC); however, the epidemiological data are inconsistent. In this retrospective case-control study, we aimed to determine whether EBV infection underlies the risk and prognosis of LSCC. The prevalence of EBV infection, as analyzed using an EBV DNA polymerase chain reaction assay, was significantly higher in 42 Taiwanese patients with newly diagnosed primary LSCC, compared to 39 age- and sex-matched control patients without cancer $(48 \%$ vs. $19 \%)$. Furthermore, most of the EBER signals detected using in situ hybridization were localized to the nuclei of tumor-infiltrating lymphocytes. In multivariate analysis, EBV DNA positivity, age $\geq 55$ years, cigarette smoking, and high BCL-2, B2M, and CD161 expression (assessed using immunohistochemistry) were identified as independent risk factors for LSCC. Furthermore, five-year local recurrence and disease-free survival rates were $34 \%$ and $58 \%$, respectively, with a high EBER signal and low CD3 expression independently predicting five-year local recurrence and disease-free survival. Our comprehensive profiling data accurately identified patients at risk for LSCC development, local recurrence, or disease-free survival. The information obtained in this study improves our understanding of EBV infection in LSCC, and may guide precision medicine for patients with LSCC. 
Keywords: $B C L-2 ; B 2 M$; CD3; CD161; Epstein-Barr virus; EBER; laryngeal squamous cell carcinoma; local recurrence; disease-free survival; risk

\section{Introduction}

The long-term survival of patients with laryngeal squamous cell carcinoma (LSCC) is excellent when locoregional recurrence is controlled [1]. However, approximately $2.0 \%$ of deaths per annum are attributable to LSCC, with survivors frequently experiencing impaired voice quality $[2,3]$. Therefore, the etiologic risk factors for primary and recurrent LSCC need to be thoroughly studied; potential risk factors include cigarette smoking, alcohol consumption, environmental carcinogens, and gene mutations [4]. According to previous studies, additional factors include human papillomavirus [4-6], contrary to our findings [7]; and Epstein-Barr virus (EBV) [8-12], contrary to various reports [5,13,14].

As the upper aerodigestive tract is in constant contact with the environment, it is easily infected by EBV, making it susceptible to local carcinogenesis [9]

In nasopharyngeal carcinoma, local infiltration of EBV-infected leukocytes creates a tumor microenvironment that promotes tumor development [15]. However, the effects of EBV infection on the host's genetic susceptibility (e.g., expression of BCL-2 (the Akt signaling pathway) [16], MYC (the TGF- $\beta$ signaling pathway) [17], and $p 16 I N K 4 a$ (the cell cycle G1/S checkpoint signaling pathway) [7]) and immune response (e.g., expression of $B 2 M$ [18], CD3 [19], and CD161 [20]) to LSCC have not been reported in detail. Moreover, the relationship between viral infections and LSCC development and prognosis requires further investigation.

In this study, we aim to examine the following hypotheses: (1) EBV infection underlies the risk of primary LSCC, and (2) EBV infection affects the prognosis of LSCC, using a more comprehensive approach. The potential mechanisms of virus-related carcinogenesis will also be discussed.

\section{Materials and Methods}

\subsection{Study Populations}

This was a retrospective case-control study. We included patients with laryngeal lesions who had undergone laryngeal surgery in the Department of Otorhinolaryngology-Head and Neck Surgery at Chang Gung Memorial Hospital, Linkou Main Branch (Taoyuan City, Taiwan) between 1 August 2012 and 31 December 2015. These patients had been recruited to a previous study exploring tumor microbiology in laryngeal cancer (funder: Chang Gung Medical Foundation; no. CMRPG3C0661-3). The primary inclusion criteria were as follows: age $>18$ years, pathological diagnosis of a laryngeal lesion, and willingness to sign informed consent forms. The exclusion criteria included an unwillingness to undergo tissue examination and/or answer subjective questionnaires. All investigations were conducted in accordance with the World Medical Association Declaration of Helsinki. This study followed the recommendations for prognostic studies of tumor biomarkers (REMARK) [21].

In this study, the case group consisted of patients with newly diagnosed, histologically confirmed LSCC. The control group consisted of patients without a history of cancer who were treated for a non-malignant lesion of the larynx (NMLL), such as polyps and leukoplakia. Patients with initial NMLLs who had malignant transformations during the study period were excluded from this study. Case patients were matched to control patients according to sex.

This study also aimed to investigate the effects of EBV infection on long-term prognoses, including five-year local recurrence (LR), neck recurrence (NR), distant metastasis (DM), disease-free survival (DFS), disease-specific survival (DSS), and overall survival (OS), of primary LSCCs. Medical records obtained from the electronic health information system until 28 February 2021 were reviewed. The study was approved by the Institutional 
Review Board (no. 202100292B0) of the Chang Gung Medical Foundation (Taoyuan City, Taiwan). Written informed consent for this study was waived.

\subsection{Clinicopathologic Evaluations}

Data on the following variables were collected at enrollment: age at diagnosis, sex, smoking status, alcohol consumption status, pathological diagnosis, pathological staging (according to the 2009 revision of the American Joint Committee on Cancer tumor-nodemetastasis staging system) [22], treatment modality, and LR. LSCC patients underwent transoral laser microsurgery (TLM), radiation therapy (RT), or concurrent chemoradiotherapy (CCRT) for T1-3 tumors, or total laryngectomy with neck dissection for T4a tumors, as previously described $[3,23,24]$. En bloc resection was attempted for all lesions, and tumorfree margins were confirmed using frozen-section analysis during the operation $[3,23,24]$. If positive primary excision margins were noted, additional extended resections were performed until all secondary margins were negative for malignancy [25]. Patients for whom complete resection was not possible, as determined by a multidisciplinary tumor board at the hospital, were suggested for adjuvant RT or CCRT [23].

In this study, LR was defined as a positive biopsy in the region of the primary tumor following a complete response to treatment, or in the area of the primary tumor following radical surgery (i.e., a negative post-treatment screen). NR was defined as a positive cytology/biopsy in the cervical lymphatic region following primary surgery. DM was identified through biopsy or imaging, as verified by our tumor board. Therefore, the five-year LR, NR, DM, DFS, DSS, and OS rates were calculated.

\subsection{Detection of EBV DNA in Tumor Tissue and Plasma}

Formalin-fixed paraffin-embedded (FFPE) tissue sections were prepared from specimens that had been histologically evaluated for lesion type and tissue adequacy $(\geq 10.0 \%$ LSCC/NMLL cells) using hematoxylin and eosin staining [26]. We have previously detailed the anti-contamination procedures elsewhere [27]. Three 5- $\mu \mathrm{m}$-thick FFPE sections per specimen were used for DNA extraction (Lab Turbo Virus Mini Kit; TaiGen Biotechnology Co., Taipei, Taiwan) [7].

Plasma DNA from $10 \mathrm{~mL}$ of peripheral blood was extracted using a QIAamp DNA Blood MiniKit (Qiagen Inc., Valencia, CA, USA). Approximately $500 \mu \mathrm{L}$ of each sample per column was eluted with $80 \mu \mathrm{L}$ of distilled water and used for DNA detection [28].

Real-time polymerase chain reaction (PCR) assays were performed using an ABI Prism 7700 Sequence Detection Analyzer (Applied Biosystems, Carlsbad, CA, USA) to detect the BamHI W fragment in the EBV genome; we previously detailed the real-time PCR protocol elsewhere [28]. Briefly, we used primers to detect the BamHI W region sequence (forward: 5'CCCAACACTCCACCACACC3'; reverse: 5'TCTTAGGAGCTGTCCGAGGG3'; and a dual fluorescence-labeled oligomer: $5^{\prime}$ [FAM]CACACACTACACACACCCACCCG TCTC[TAMRA] $3^{\prime}$ ) [29]. EBV DNA positivity was defined as a detectable EBV-DNA signal after 40 cycles of PCR, whereas EBV DNA negativity was defined as a copy number of zero [30].

\subsection{Detection of EBV Antibody in Serum}

Serum obtained from $3 \mathrm{~mL}$ of peripheral blood was used to determine serum EBV titers. An immunofluorescence assay specific for EBV viral capsid antigen (VCA) IgA (Meridian Bioscience Inc., Cincinnati, OH, USA) was performed in accordance with the manufacturer's protocol. We have previously outlined the immunofluorescence assay protocol elsewhere [31]. Briefly, acetone-fixed B95.8 cell glass slides were applied to dilutions of patient sera. Appropriate concentrations of fluorescein isothiocyanate-conjugated anti-human IgA were applied following incubation and washing. The antibody titer of the test serum was defined as the reciprocal of the highest dilution that clearly showed apple-green fluorescence in $20 \%$ of cells. The cut-off values for EBV VCA IgA were set at 1:40 [31]. 


\subsection{Tissue Microarray Construction}

All routine hematoxylin and eosin-stained sections of FFPE tissue blocks that had been histologically evaluated for lesion type and tumor tissue adequacy were used for tissue microarray (TMA) construction, the protocol of which is described elsewhere $[7,25]$. Briefly, two regions within LSCC or NMLL foci per case were marked on hematoxylin and eosin-stained slides by a pathologist (TCC) blinded to the patient's clinical information and used to assemble the recipient blocks. Tissue biopsy cores, each $1.0 \mathrm{~mm}$ in diameter and $4 \mu \mathrm{m}$ in thickness, were taken from corresponding donor blocks.

\subsection{In Situ Hybridization}

For in situ hybridization (ISH) detection of EBV-encoded small RNAs (EBERs), biotinlabeled EBV probe solutions (Bond Ready-to-Use EBER Probe, cat. \# PB0589; Leica Biosystems Ltd., Newcastle, UK) were applied to TMA sections using an automated immunostainer (Bond-Max; Leica Microsystems $\mathrm{GmbH}$, Wetzlar, Germany), according to the manufacturer's instructions. Tumor tissue from EBV-positive nasopharyngeal carcinoma (NPC) was used as a positive control, while LSCC tissue from EBV-negative samples was used as a negative control. As EBERs are expressed in tumor-infiltrating lymphocytes (TILs), neighboring lymphocytes, and tumor cells [32], EBER ISH was considered "positive" when $E B E R$ staining was present in tumor cells and/or peritumoral stroma.

\subsection{Immunohistochemistry Staining}

Immunohistochemistry (IHC) was used to detect the expression of BCL-2 (cat. \# M088701; Dako North America, Inc., Carpinteria, CA, USA), MYC (cat. \# Z2258; Zeta Corp., Sierra Madre, CA, USA), p16INK4a (cat. \# E6H4; Roche Diagnostics GmbH, Heidelberg, Germany), B2M (cat. \# NCL-B2MP; Leica Biosystems Ltd., Newcastle, UK), CD3 (cat. \#s NCL-L-CD3-565; Leica Biosystems Ltd., Newcastle, UK), and CD161 (cat. \# orb5815; Bioorbyt, Cambridge, UK). TMA tissue sections were stained using the same immunostains noted above, according to the manufacturer's instructions. Detailed IHC procedures have been previously described [7,25]. For optimal detection, antibodies were diluted at the following ratios: 1:10 (p16INK4a), 1:500 (BCL-2), 1:50 (MYC), 1:100 (B2M), 1:200 (CD3), and 1:100 (CD161). Antibody reactions were performed at room temperature for $20 \mathrm{~min}$.

\subsection{Computer-Supported Evaluation}

Stained slides were digitalized at $40 \times$ magnification using an Aperio ScanScope scanner (Leica Biosystems, Richmond, IL, USA). Expression levels of EBER and histological marker proteins were digitally assessed in four specified regions of interest (ROIs), which contained the laryngeal mucosal lesion and submucosal tissue, using semi-quantitative image analysis (Tissue Studio v2.1; Definiens AG, Münich, Germany) $[7,25,33]$. The image analysis software allowed for the specific mining of staining intensities in the cell nuclei (EBER and MYC) or whole cells ( $p 16 I N K 4 a, B C L-2, B 2 M, C D 3$, and CD161); nuclei and cells were categorized as "negative" or "positive" (weak, moderate, or high staining intensity). The positivity index (PI; \%) ((number of positively stained cells or nuclei/total number of cells or nuclei) $\times 100$ ) was calculated. The same investigator who was blinded to the clinical outcomes chose the ROIs and performed a computer-supported evaluation.

\subsection{Sample Size Calculation}

Sample sizes were estimated according to our prior study (Fisher's exact test, twotailed $\alpha=0.05$, power $=0.80$; allocation ratio $=1$ ), which was performed in a previous study investigating EBV infections in patients with LSCC (52\%), vocal cord leukoplakia, and polyps $(20 \%)$. A total of at least 80 patients were required to achieve the aims of the study. 


\subsection{Statistical Analysis}

As most variables were not normally distributed, as assessed using the KolmogorovSmirnov test, data were compared using the Mann-Whitney $U$, Kruskal-Wallis, chi-square, or Fisher's exact test, as appropriate.

The odds ratio (OR) of each covariate was derived from a simple or multiple logistic regression model as an estimated coefficient with a 95\% confidence interval (CI). Spearman correlation coefficients were computed between selected predictors. For translational purposes, an optimal cut-off value was used to further dichotomize selected continuous variables using receiver operating characteristic curves with Youden's J statistic as the best trade-off between sensitivity and specificity, to predict LSCC [34].

Survival curves were plotted using the Kaplan-Meier method and compared using the log-rank test. Optimal cut-off values were used to further dichotomize selected continuous variables using time-dependent receiver operating characteristic curves [35] with Youden's $\mathrm{J}$ statistic as the best trade-off between sensitivity and specificity, to predict the prognoses. Furthermore, hazard ratios (HRs) and their corresponding 95\% CIs were calculated.

Variables with $p<0.05$ in univariate analyses were entered into multivariate logistic regression models for risk analysis, or Cox regression models for prognosis analysis. Twotailed $p$-values $<0.05$ were considered statistically significant.

Statistical analyses were conducted using R (version 3.6.1, R Foundation for Statistical Computing, http:/ / www.r-project.org/, accessed on 28 February 2021) and GraphPad Prism for Windows software (version 9.0, GraphPad Software Inc., San Diego, CA, USA).

\section{Results}

\subsection{Clinical Characteristics of Patients}

A total of 98 patients were identified, 17 of whom were excluded due to insufficient tissues $(n=14)$ and low biomarker quality $(n=3)$ (Figure 1$)$. Therefore, the overall cohort comprised of 42 patients with LSCC (cases) and 39 patients with NMLL (controls); a total of 81 patients $(74(91 \%)$ men and $7(9 \%)$ women) with a median age of 58 years (interquartile age: 51-70 years). Table 1 summarizes the baseline characteristics of the populations in the study. Age $\geq 55$ years (unadjusted $\mathrm{OR}=8.3 ; 95 \%$ CI: 3.0-22.5; $p<0.001$ ) and cigarette smoking (unadjusted $\mathrm{OR}=4.5 ; 95 \% \mathrm{CI}$ : $1.1-17.8 ; p=0.03$ ) were significantly associated with a risk of LSCC.

Table 1. Clinicopathological characteristics and treatments.

\begin{tabular}{|c|c|c|c|c|}
\hline Characteristic & Overall & Patients with LSCC & Patients with NMLL & $p$-Value ${ }^{\mathrm{a}}$ \\
\hline \multicolumn{5}{|c|}{ Clinical Characteristics } \\
\hline Patients & $n=81$ & $n=42$ & $n=39$ & \\
\hline Male, $n(\%)$ & $74(91)$ & $38(91)$ & $36(92)$ & \multirow{2}{*}{$>0.99$} \\
\hline Female, $n(\%)$ & $7(9)$ & $4(9)$ & $3(8)$ & \\
\hline Age (years), median (range) & $58(51-70)$ & $64(58-74)$ & $52(37-58)$ & $<0.001$ \\
\hline Age $\geq 55$ years, $n(\%) b$ & $45(56)$ & $33(79)$ & $12(31)$ & \multirow{2}{*}{$<0.001$} \\
\hline Age $<55$ years, $n(\%) b$ & $36(44)$ & $9(21)$ & $27(69)$ & \\
\hline Cigarette smoker, $n(\%)$ & $69(85)$ & $39(93)$ & $29(74)$ & \multirow{2}{*}{0.03} \\
\hline Never smoker, $n(\%)$ & $12(15)$ & $3(7)$ & $10(26)$ & \\
\hline Alcohol drinker, $n(\%)$ & $48(59)$ & $27(64)$ & $21(54)$ & \multirow{2}{*}{0.37} \\
\hline Never drinker, $n(\%)$ & $33(41)$ & $15(36)$ & $18(46)$ & \\
\hline \multicolumn{5}{|c|}{ Pathological Characteristics } \\
\hline Polyps, $n(\%)$ & - & - & $18(46)$ & - \\
\hline Leukoplakia, $n(\%)$ & - & - & $21(54)$ & - \\
\hline
\end{tabular}


Table 1. Cont.

\begin{tabular}{ccccc}
\hline Characteristic & Overall & Patients with LSCC & Patients with NMLL & $\boldsymbol{p}_{\text {-Value }}{ }^{\text {a }}$ \\
\hline T1-2, $n(\%)$ & - & $34(81)$ & - & - \\
T3-4, $n(\%)$ & - & $8(19)$ & - & - \\
\hline N0, $n(\%)$ & - & $40(95)$ & - & - \\
N1-2, $n(\%)$ & - & $2(5)$ & - & - \\
Stage I-II, $n(\%)$ & - & $34(81)$ & - & - \\
Stage III-IV, $n(\%)$ & - & $8(19)$ & & - \\
\hline
\end{tabular}

Treatment Modalities

\begin{tabular}{ccccc}
\hline Transoral laser microsurgery, $n(\%)$ & - & $28(67)$ & - & - \\
Total laryngectomy, $n(\%)$ & & $3(7)$ & - & - \\
RT, $n(\%)$ & - & $4(10)$ & - & - \\
CCRT, $n(\%)$ & - & $3(7)$ & - & - \\
Surgery + RT, $n(\%)$ & - & $3(7)$ & - & - \\
Surgery + CCRT, $n(\%)$ & & $1(2)$ & - & - \\
\hline Single-modality treatment, $n(\%)$ & - & $31(74)$ & - & - \\
Multiple-modality treatment, $n(\%)$ & - & $11(26)$ & \\
\hline
\end{tabular}

Abbreviations: CCRT, concurrent chemoradiotherapy; LSCC, laryngeal squamous cell carcinoma; NMLL, non-malignant laryngeal lesion; RT, radiotherapy. ${ }^{a}$ Data were compared between LSCC and NMLL subgroups using the Mann-Whitney $U$ test or Fisher's exact test, as appropriate. ${ }^{b}$ The optimal cut-off value using receiver operating characteristic curves with Youden's J-point as the best trade-off between sensitivity and specificity, to predict LSCC. Significant $p$-values are marked in bold.

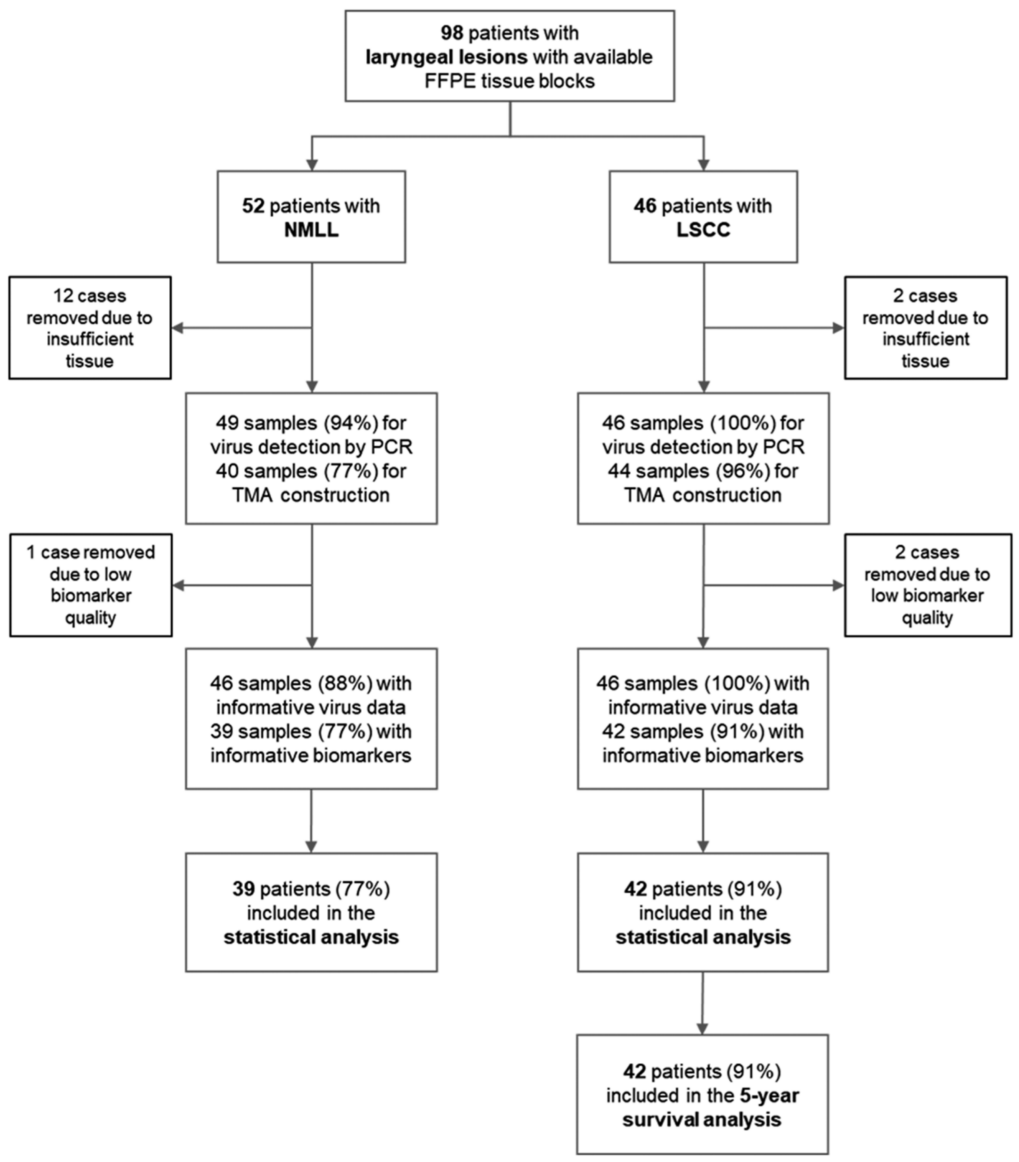

Figure 1. REMARK diagram. There were 94 patients with laryngeal lesions with available formalinfixed paraffin-embedded (FFPE) tissue blocks. Seventeen cases with inadequate tissue and lowquality biomarkers were excluded. Therefore, 42 patients with laryngeal squamous cell carcinoma (LSCC) and 39 sex-matched control patients with a non-malignant lesion of the larynx (NMLL) were included in the final statistical analyses. 
Most patients had early stage (Stage I-II) LSCC (81\%) and had undergone transoral laser microsurgery $(67 \%)$ or RT $(10 \%)$ as the definitive treatment.

\subsection{EBV-Related Biomarkers in the Larynx and Peripheral Blood}

The overall laryngeal EBV infection rate was 37\% (Table 2). The laryngeal EBV DNA positivity rate was significantly higher in case, compared to control, patients (52\% vs. $20 \%$; effect size $=0.70 ; 95 \%$ CI $0.24-1.16 ; p=0.01$ ); therefore, patients with laryngeal EBV DNA positivity had a greater risk of developing LSCC, compared to those with EBV DNA negativity (unadjusted OR $=4.3 ; 95 \%$ CI: $1.6-11.4 ; p=0.004$ ).

Table 2. EBV status in laryngeal tissue and peripheral blood.

\begin{tabular}{|c|c|c|c|c|}
\hline Characteristics & Overall & Patients with LSCC & Patients with NMLL & $p$-Value ${ }^{a}$ \\
\hline \multicolumn{5}{|c|}{ Laryngeal Tissue } \\
\hline Patients & $n=81$ & $n=42$ & $n=39$ & \\
\hline EBV DNA positivity, $n(\%)$ & $30(37)$ & $22(52)$ & $8(20)$ & \multirow[b]{2}{*}{0.01} \\
\hline EBV DNA negativity, $n(\%)$ & $51(63)$ & $20(48)$ & $31(80)$ & \\
\hline $\mathrm{nPI}$ for $E B E R,(\%)$, median (range) & $0.02(0.01-0.06)$ & $0.03(0.01-0.05)$ & $0.02(0.01-0.07)$ & $<0.001$ \\
\hline$E B E R$ signal $\geq 0.02 \%, n(\%) \mathrm{b}$ & $50(62)$ & $28(67)$ & $22(56)$ & \multirow{2}{*}{0.37} \\
\hline EBER signal $<0.02 \%, n(\%){ }^{b}$ & $31(62)$ & $14(33)$ & $17(44)$ & \\
\hline \multicolumn{5}{|c|}{ Peripheral Blood } \\
\hline Patients & - & $n=30$ & - & \\
\hline Circulating EBV DNA positivity, $n(\%)$ & - & $2(7)$ & - & - \\
\hline Circulating EBV DNA negativity, $n(\%)$ & - & $28(93)$ & - & - \\
\hline EBV-VCA IgA positivity, $n(\%)$ & - & $4(13)$ & - & - \\
\hline EBV VCA IgA negativity, $n(\%)$ & - & $26(87)$ & - & - \\
\hline
\end{tabular}

Abbreviations: EBER, Epstein-Barr encoding region; EBV, Epstein-Barr virus; LSCC, laryngeal squamous cell carcinoma; NMLL, nonmalignant laryngeal lesion; VCA, viral capsid antigen; PI, positivity index. ${ }^{a}$ Data were compared between LSCC and NMLL subgroups using the Mann-Whitney $U$ test or Fisher's exact test, as appropriate. ${ }^{b}$ The optimal cut-off value using receiver operating characteristic curves with the Youden's J-point as the best trade-off between sensitivity and specificity, to predict LSCC. Significant results with their $p$-values are marked in bold.

The laryngeal nPI for EBER was relatively low in both case and control groups, and the staining distribution was equal between the groups (Table 2). Most $E B E R$ signals were localized to the nuclei of TILs (Figure 2a); however, laryngeal EBV DNA positivity was not related to laryngeal nPI for EBER $(r=0.11, p=0.34)$.

Only 30 patients with LSCC had available peripheral blood EBV test data; among these patients, two $(7 \%)$ and four $(13 \%)$ had circulating EBV DNA positivity and serum EBV VCA IgA positivity, respectively. Differences in circulating EBV DNA positivity (7\% vs. $6 \%, p>0.99)$ and EBV VCA IgA positivity ( $7 \%$ vs. $19 \%, p=0.60)$ between laryngeal EBV DNA-positive and -negative subgroups were not statistically significant. Therefore, the associations between laryngeal EBV DNA positivity, laryngeal nPI for EBER, plasma EBV DNA positivity, and serum EBV VCA IgA positivity were not statistically significant (all $p>0.05$ ). 
(a) $E B E R$

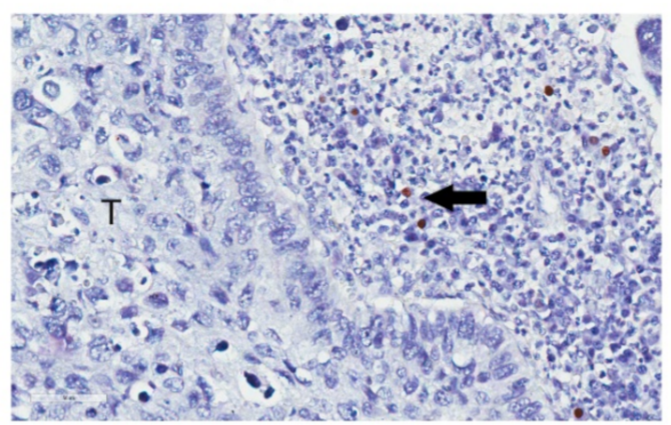

(b) $B C L-2$

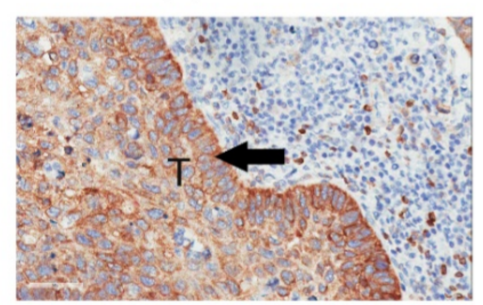

(e) $B 2 M$

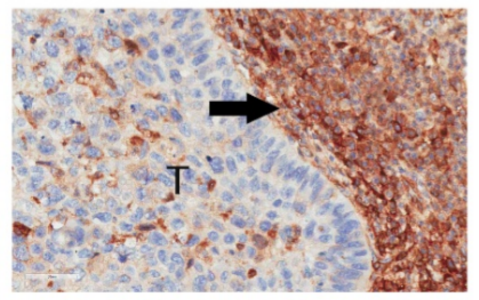

(c) $M Y C$

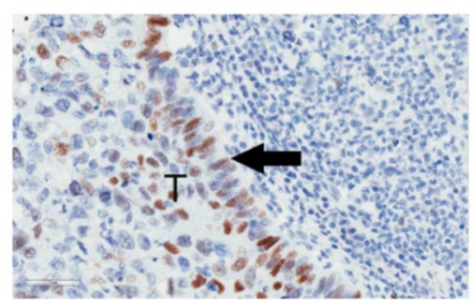

(f) $\mathrm{CD} 3$

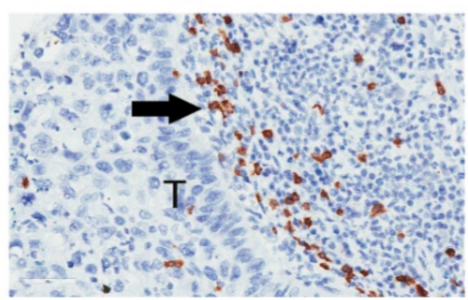

(d) $p 16 I N K 4 a$

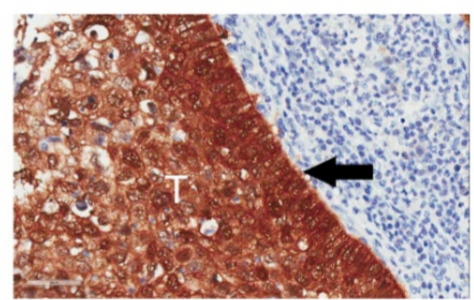

(g) CD161

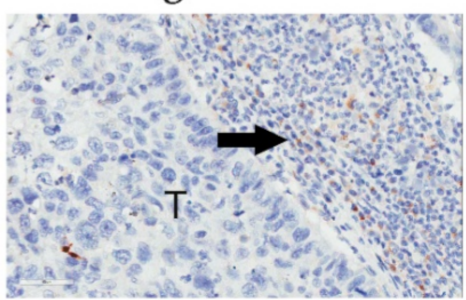

Figure 2. Evaluation of Epstein-Barr virus-encoding RNA (EBER), BCL-2, MYC, p16INK4a, B2M, CD3, and CD161 in a representative case with laryngeal squamous cell carcinoma. (a) EBER signals (arrow) were detected using in situ hybridization in the nuclei of tumor-infiltrating lymphocytes. Expression of tumor markers, including (b) BCL-2, (c) MYC, and (d) p16INK4a (arrows), occurred predominantly in intratumoral cells, whereas expression of immunological markers, including (e) B2M, (f) CD3, and (g) CD161 (arrows), occurred mainly in peritumoral cells. Original magnification $40 \times$.

\subsection{Histological Factors of the Larynx}

In the IHC analysis, expression of $p 16 I N K 4 A, B C L-2$, and MYC occurred mainly in intratumoral cells (Figure $2 \mathrm{~b}-\mathrm{d}$ ), whereas $B 2 M, C D 3$, and $C D 161$ expression occurred mainly in peritumoral (Figure 2e-g) cells. Expression of BCL-2, B2M, CD3, and CD161 was higher, MYC was lower, and $p 16 I N K 4 a$ was the same in LSCC, compared with NMLL (Table 3).

We further dichotomized these biomarkers using receiver operating characteristic curve analyses, with Youden's J-point as the best trade-off between sensitivity and specificity, to predict LSCC. Expression of $B C L-2 \geq 68.6 \%$ (unadjusted OR $=5.5 ; 95 \%$ CI: 2.0-14.9; $p=0.001$ ), $B 2 M \geq 84.3 \%$ (unadjusted $\mathrm{OR}=8.6 ; 95 \%$ CI: $3.1-23.9 ; p<0.004$ ), $C D 3 \geq 6.9 \%$ (unadjusted OR $=7.2 ; 95 \%$ CI: $2.6-20.2 ; p<0.001$ ), CD161 $\geq 68.8 \%$ (unadjusted OR = 3.5; 95\% CI: 1.3-9.4; $p=0.01$ ), MYC $\leq 0.44 \%$ (unadjusted OR $=2.9 ; 95 \%$ CI 1.2-7.1; $p=0.02$ ), and $p 16 I N K 4 a \leq 16.8 \%$ (unadjusted OR $=6.0 ; 95 \%$ CI 1.6-22.9; $p=0.01$ ) were significant risk factors for LSCC. 
Table 3. Histological factors of the larynx.

\begin{tabular}{|c|c|c|c|c|}
\hline Characteristics & Overall & Patients with LSCC & Patients with NMLL & $p$-Value $^{\mathrm{a}}$ \\
\hline \multicolumn{5}{|c|}{ Tumor-Related Biomarkers in the Larynx } \\
\hline Patients & $n=81$ & $n=42$ & $n=39$ & \\
\hline cPI for BCL-2, (\%), median (range) & $82.9(43.3-98.2)$ & $86.7(76.8-98.7)$ & $61.5(35.5-94.2)$ & 0.01 \\
\hline$B C L-2$ expression $\geq 68.6 \%, n(\%) b$ & $30(37)$ & $34(81)$ & $17(44)$ & \multirow{2}{*}{0.001} \\
\hline$B C L-2$ expression $<68.6 \%, n(\%){ }^{b}$ & $51(63)$ & $8(19)$ & $22(56)$ & \\
\hline nPI for $M Y C,(\%)$, median (range) & $0.44(0.24-0.56)$ & $0.38(0.17-0.53)$ & $0.50(0.32-0.67)$ & 0.03 \\
\hline MYC expression $\leq 0.44 \%, n(\%) b$ & $44(54)$ & $28(67)$ & $16(41)$ & \multirow{2}{*}{0.03} \\
\hline$M Y C$ expression $>0.44 \%, n(\%){ }^{b}$ & $37(46)$ & $14(33)$ & $23(59)$ & \\
\hline cPI for $p 16 I N K 4 a,(\%)$, median (range) & $49.8(20.3-93.8)$ & $45.3(11.5-92.4)$ & $49.9(25.0-96.3)$ & 0.21 \\
\hline$p 16 I N K 4 a$ expression $\leq 16.8 \%, n(\%) b$ & $17(21)$ & $14(33)$ & $3(8)$ & \multirow{2}{*}{0.01} \\
\hline$p 16 I N K 4 a$ expression $>16.8 \%, n(\%){ }^{b}$ & $64(79)$ & $28(67)$ & $36(92)$ & \\
\hline \multicolumn{5}{|c|}{ Host Mucosal Immune-Related Biomarkers in the Larynx } \\
\hline cPI for $B 2 M,(\%)$, median (range) & $82.9(68.2-96.3)$ & 92.5 (79.5-97.6) & $74.1(55.9-84.0)$ & 0.001 \\
\hline$B 2 M$ expression $\geq 84.3 \%, n(\%)^{b}$ & $37(46)$ & $29(69)$ & $8(21)$ & \multirow{2}{*}{$<0.001$} \\
\hline$B 2 M$ expression $<84.3 \%, n(\%) b$ & $44(54)$ & $13(31)$ & $31(79)$ & \\
\hline cPI for CD3, (\%), median (range) & $11.3(3.8-27.3)$ & $20.5(9.1-32.8)$ & $6.3(2.0-15.4)$ & 0.001 \\
\hline CD3 expression $\geq 6.9 \%, n(\%)^{b}$ & $51(63)$ & $35(83)$ & $16(41)$ & \multirow{2}{*}{$<0.001$} \\
\hline CD3 expression $<6.9 \%, n(\%){ }^{b}$ & $30(37)$ & 7 (17) & $23(59)$ & \\
\hline cPI for CD161, $(\%)$, median (range) & $47.9(14.7-76.9)$ & $59.8(23.5-80.9)$ & $32.0(6.7-67.3)$ & 0.004 \\
\hline CD161 expression $\geq 68.8 \%, n(\%)$ & $28(35)$ & $20(48)$ & $8(21)$ & \multirow[b]{2}{*}{0.02} \\
\hline CD161 expression $<68.8 \%, n(\%)$ b & $53(65)$ & $22(52)$ & $31(80)$ & \\
\hline
\end{tabular}

Abbreviations: EBER, Epstein-Barr encoding region; EBV, Epstein-Barr virus; LSCC, laryngeal squamous cell carcinoma; NMLL, nonmalignant laryngeal lesion; VCA, viral capsid antigen; PI, positivity index. ${ }^{a}$ Data were compared between LSCC and NMLL subgroups using the Mann-Whitney $U$ test or Fisher's exact test, as appropriate. ${ }^{b}$ The optimal cut-off value using receiver operating characteristic curves with Youden's J-point as the best trade-off between sensitivity and specificity, to predict LSCC. Significant results with their $p$-values are marked in bold.

3.4. Relationship between EBV-Related Biomarkers and Clinical and Histological Characteristics in the Overall Cohort

In the overall cohort, primary LSCC was significantly associated with age $\geq 55$ years, cigarette smoking, EBV DNA positivity, BCL-2 expression $\geq 68.6 \%, B 2 M$ expression $\geq$ $84.3 \%, C D 3$ expression $\geq 6.9 \%, C D 161$ expression $\geq 68.8 \%, M Y C$ expression $\leq 0.44 \%$, and p16INK4a expression $\leq 16.8 \%$ (Table 4 ).

Additionally, the male sex was significantly associated with cigarette smoking and alcohol consumption. The associations between age $\geq 55$ years and MYC expression $\leq$ $0.44 \%, B 2 M$ expression $\geq 84.3 \%$, and $C D 3$ expression $\geq 6.9 \%$ were significant. Furthermore, the relationship between cigarette smoking and alcohol consumption was significant.

EBV DNA positivity was significantly correlated with high $B C L-2$ expression ( $\geq 68.6 \%$ ), which also significantly correlated with $B 2 M$ expression $\geq 84.3 \%, C D 3$ expression $\geq 6.9 \%$, and $C D 161$ expression $\geq 68.8 \%$. Low $p 16 I N K 4 a$ expression $(\leq 16.8 \%)$ was significantly associated with $B 2 M$ expression $\geq 84.3 \%$ and $C D 161$ expression $\geq 68.8 \%$. B2M expression $\geq 84.3 \%$ was significantly associated with $C D 3$ expression $\geq 6.9 \%$, which also significantly associated with CD161 expression $\geq 68.8 \%$. 
Table 4. Spearman's correlations between clinical characteristics, laryngeal EBV-related biomarkers, and laryngeal histological characteristics in the overall cohort.

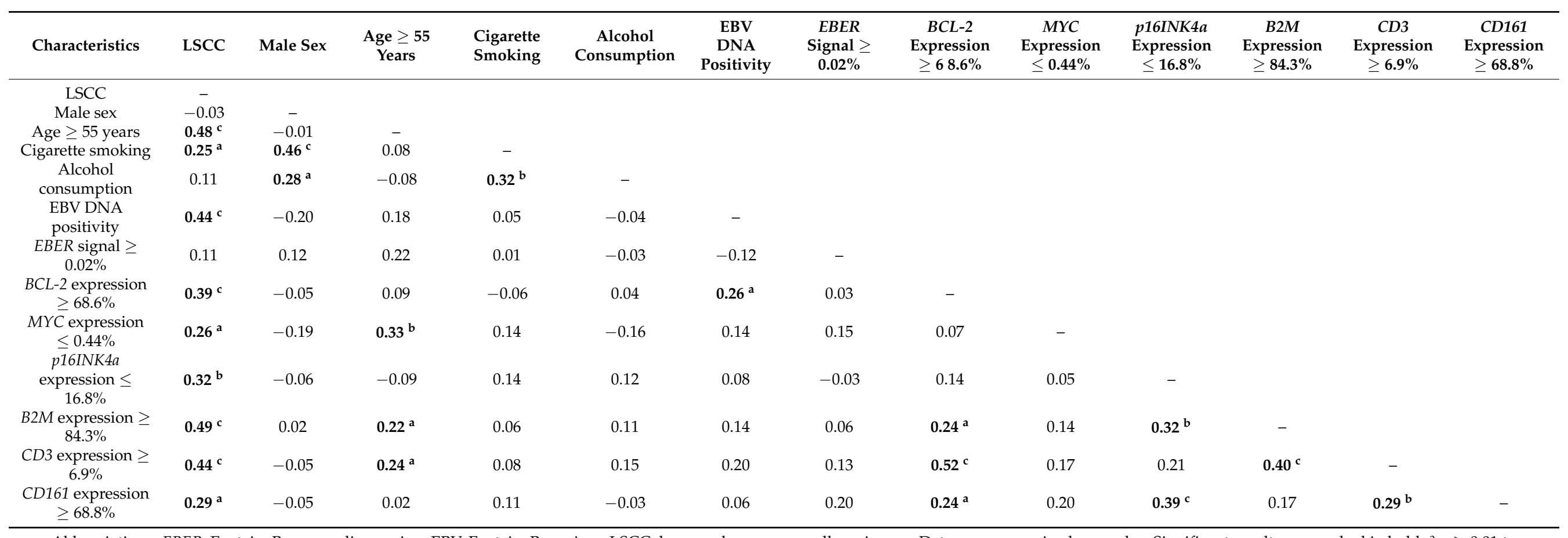

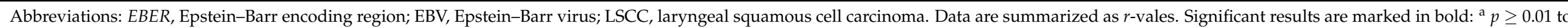
$<0.05,{ }^{\mathrm{b}} p \geq 0.001$ to $<0.01$, and ${ }^{\mathrm{c}} p<0.001$. 


\subsection{Multi-Factor Modeling of Primary LSCC}

To improve the precision of the model, we used a "forced simultaneous entry" approach and a "sign-correct" method for multivariate logistic regression analysis [36]. Variables with a $p<0.05$ in the univariate analysis were entered into the regression analysis. Age $\geq 55$ years $(\mathrm{OR}=19.3 ; 95 \% \mathrm{CI}: 3.2-116.0 ; p=0.001)$, cigarette smoking $(\mathrm{OR}=36.4$; 95\% CI: 2.4-554.2; $p=0.01$ ), EBV DNA positivity (OR $=39.7 ; 95 \%$ CI: 3.3-478.0; $p=0.004$ ), $B C L-2$ expression $\geq 68.6 \%(\mathrm{OR}=6.8 ; 95 \%$ CI: $1.2-38.6 ; p=0.03), B 2 \mathrm{M}$ expression $\geq 84.3 \%$ $(\mathrm{OR}=18.1 ; 95 \% \mathrm{CI}: 2.9-11.6 ; p=0.002)$, and $C D 161$ expression $\geq 68.8 \%(\mathrm{OR}=7.5 ; 95 \% \mathrm{CI}$ : $1.1-50.7 ; p=0.04)$ were significant independent risk factors for primary LSCC.

Using this six-factor model, we accurately predicted which patients with laryngeal lesions had primary LSCC via the optimal cut-off value of 3 (area under the receiver operating characteristic curve: 0.87 [95\% CI: 0.79-0.96]; $p<0.001$ ), with a sensitivity, specificity, positive predictive value, and negative predictive value of $98 \%, 77 \%, 82 \%$, and $97 \%$, respectively.

\subsection{Five Year Prognoses in Patients with LSCC}

As of 28 February 2021, the median follow-up time was 68 months (range: 9-94 months). A total of 14 case patients had an LR within the first 5 years after definitive treatment; thus, the five-year LR rate was 34\% (95\% CI: 16-53\%). Two patients had NR (five-year NR rate = 6\%; $95 \%$ CI: $0-50 \%$ ) and one had DM (five-year DM rate $=3 \%$; $95 \%$ CI: 0-59\%); therefore, five-year DFS was 58\% (95\% CI: 41-71\%). Furthermore, two patients had disease-specific deaths (five-year DSS rate $=94 \%$; 95\% CI: 75-98\%), while another two died of other causes (five-year OS rate $=89 \%$; 95\% CI: 72-96\%).

Herein, we further focused on investigating the risk factors associated with five-year LR and DFS rates (Table 5). EBV DNA positivity did not significantly predict five-year LR and DFS rates. However, an EBER signal $\geq 0.04 \%$ significantly predicted both the five-year LR (unadjusted HR $=6.2 ; 95 \%$ CI: $2.0-18.6 ; p=0.001$ ) and DFS (unadjusted HR = 7.1; 95\% CI: 2.6-19.5; $p<0.001$ ) rates using univariate Cox regression models. Furthermore, age $\leq 63$ years, TLM, and CD3 expression $\leq 4.9 \%$ also predicted the five-year LR rate, whereas TLM, BCL-2 expression $\leq 96.0 \%, C D 3$ expression $\leq 4.9 \%$, and EBER signals $\geq$ $0.04 \%$ predicted five-year DFS rates.

Using multivariate Cox regression models, both EBER signals $\geq 0.04 \%$ (adjusted HR: 6.0; 95\% CI: $1.9-18.6 ; p=0.002$ ) and CD3 expression $\leq 4.9 \%$ (adjusted HR $=6.9 ; 95 \%$ CI: $1.9-$ 24.6; $p=0.003$ ) independently predicted five-year LR rates; both risk factors significantly predicted five-year LR rates after adjustment for treatment modality (adjusted HR $=4.7$ (95\% CI: 1.5-15.0) and 5.4 (95\% CI: 1.5-19.4), respectively; $p=0.01$, and 0.01, respectively). Similarly, both EBER signals $\geq 0.04 \%$ (adjusted HR $=8.6$; 95\% CI: 2.9-25.3; $p<0.001$ ) and $C D 3$ expression $\leq 4.9 \%$ (adjusted HR $=6.6 ; 95 \%$ CI: 1.9-23.6; $p=0.004$ ) independently predicted five-year DFS rates; these risk factors remained significant after adjustment for treatment modality (adjusted HR $=7.7$ (95\% CI: 2.5-24.0) and 5.4 (95\% CI: 1.5-19.3), respectively; $p<0.001$ and 0.01 , respectively). 
Table 5. Cox regression models of variables of interest for predicting five-year prognosis in patients with LSCC.

\begin{tabular}{|c|c|c|c|c|c|c|c|c|c|c|}
\hline \multirow{3}{*}{ Characteristics } & \multicolumn{5}{|c|}{ 5-Year Local Recurrence } & \multicolumn{5}{|c|}{ 5-Year Disease-Free Survival } \\
\hline & \multirow{2}{*}{ Cut-Off Value } & \multicolumn{2}{|c|}{ Univariate Models } & \multicolumn{2}{|c|}{ Multivariate Models } & \multirow{2}{*}{ Cut-Off Value } & \multicolumn{2}{|c|}{ Univariate Models } & \multicolumn{2}{|c|}{ Multivariate Models } \\
\hline & & HR $(95 \%$ CI) & $p$-Value & HR $(95 \% \mathrm{CI})$ & $p$-Value & & HR $(95 \%$ CI) & $p$-Value & HR $(95 \%$ CI) & $p$-Value \\
\hline Age, years & $\leq 63$ & $3.2(1.0-10.3)$ & 0.048 & - & NS & $\leq 63$ & $1.8(0.7-4.8)$ & 0.23 & & \\
\hline Cigarette smoking & yes & $23.2(0.1-82375.3)$ & 0.45 & & & yes & $23.6(0.1-29976.8)$ & 0.39 & & \\
\hline Alcohol drinking & yes & $2.2(0.6-7.9)$ & 0.23 & & & yes & $2.0(0.6-6.1)$ & 0.23 & & \\
\hline T-stage & $\leq 2$ & $30.7(0.2-6190.3)$ & 0.21 & & & $\leq 3$ & $32.8(0.3-3521.6)$ & 0.14 & & \\
\hline pStage & $\leq 2$ & $30.7(0.2-6190.3)$ & 0.21 & & & $\leq 2$ & $32.8(0.3-3521.6)$ & 0.14 & & \\
\hline Treatment & $\overline{T L M}$ & $8.3(1.1-63.8)$ & 0.04 & - & NS & TLM & $5.6(1.3-24.9)$ & 0.02 & - & NS \\
\hline EBV DNA positivity & yes & $0.56(0.2-1.64)$ & 0.28 & & & yes & $0.4(0.1-1.1)$ & 0.08 & & \\
\hline EBER signal, \% & $\geq 0.04$ & $6.2(2.0-18.6)$ & 0.001 & $6.0(1.9-18.6)$ & 0.002 & $\geq 0.04$ & $7.1(2.6-19.5)$ & $<0.001$ & $8.6(2.9-25.3)$ & $<0.001$ \\
\hline$B C L-2$ expression, $\%$ & $\leq 96.0$ & $2.9(0.8-10.6)$ & 0.10 & & & $\leq 96.0$ & $3.9(1.1-13.6)$ & 0.03 & - & NS \\
\hline MYC expression, \% & $\leq 0.50$ & $3.1(0.7-13.8)$ & 0.14 & & & $\leq 0.50$ & $4.2(1.0-18.6)$ & 0.06 & & \\
\hline p16INK4a expression, $\%$ & $\leq 81.6$ & $1.1(0.4-3.4)$ & 0.83 & & & $\leq 81.6$ & $2.1(0.7-6.0)$ & 0.16 & & \\
\hline$B 2 M$ expression, $\%$ & $\leq 92.7$ & $3.5(1.0-12.7)$ & 0.05 & & & $\leq 93.8$ & $2.0(0.7-5.7)$ & 0.19 & & \\
\hline CD3 expression, $\%$ & $\leq 4.9$ & $7.2(2.2-1209)$ & 0.001 & $6.9(1.9-24.6)$ & 0.003 & $\leq 4.9$ & $6.7(2.1-21.8)$ & 0.001 & $6.6(1.9-23.6)$ & 0.004 \\
\hline
\end{tabular}

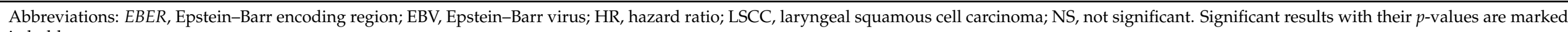
in bold. 
3.7. Relationship between EBV-Related Biomarkers and Clinical and Histological Characteristics in Patients with LSCC

In patients with primary LSCC, male sex was significantly associated with cigarette smoking and laryngeal EBV DNA positivity (Table 6). Furthermore, an age $\leq 63$ years was associated with alcohol consumption and $p 16 I N K 4 a \leq 81.6 \%$, while cigarette smoking was related to alcohol consumption. As expected, T- and N-stages were associated with LSCC stage. EBV DNA positivity and EBER signal $\geq 0.04 \%$ were not associated with other tissue biomarkers. $B C L-2$ expression $\leq 96.0 \%$ correlated significantly with $p 16 I N K 4 a$ and CD161 expression $\leq 81.6 \%$ and $\leq 69.9 \%$, respectively. In addition, $p 16 I N K 4 a$ expression $\leq 81.6 \%$ was associated with CD3 expression $\leq 4.9 \%$.

The overall cohort was further dichotomized into a high-risk group ( $\geq 1$ risk factor) and a low-risk group ( $<1$ risk factor) to predict five-year LR (sensitivity, 79\%; specificity, 94\%; positive predictive value, $88 \%$; negative predictive value, $90 \%$ ). This method performed well in predicting five-year DFS (sensitivity, 76\%; specificity, 100\%; positive predictive value, $100 \%$; negative predictive value, 52\%). The five-year LR rate of the high-risk group was significantly higher than that of the low-risk group (75\% (95\% CI, 57-86\%) vs. $11 \%$ $(95 \%$ CI, 0-49\%); $p<0.001$ ) (Figure 3a). As expected, the five-year DFS rate of the highrisk group was significantly higher than that of the low-risk group (0\% vs. 84\% (95\% CI, $63-84 \%) ; p<0.001$ ) (Figure 3b). However, this model predicted the five-year DSS (83\% $(95 \% \mathrm{CI}, 47-96 \%)$ vs. $100 \% ; p=0.05)$ and OS (76\% (95\% CI, 43-92\%) vs. $96 \%(95 \% \mathrm{CI}$, $72-99 \%) ; p=0.08$ ) rates less efficiently due to relatively low numbers of disease-specific and overall deaths.

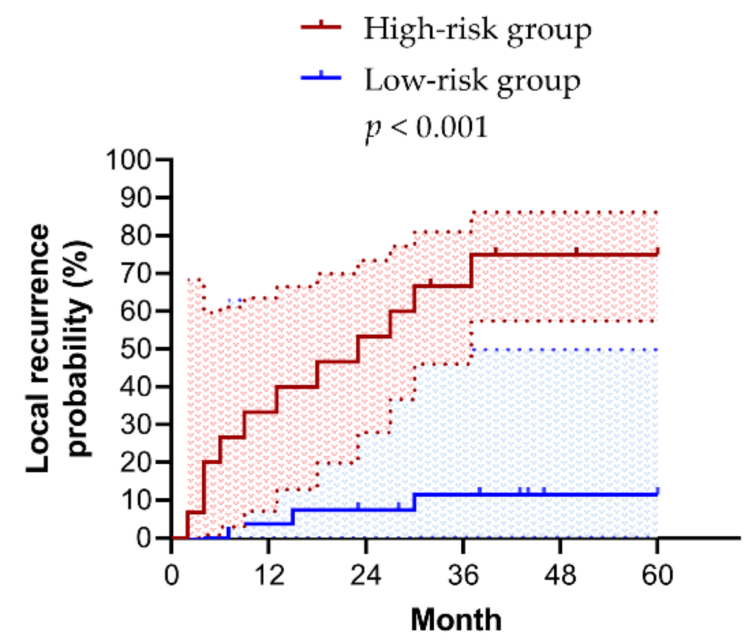

(a)

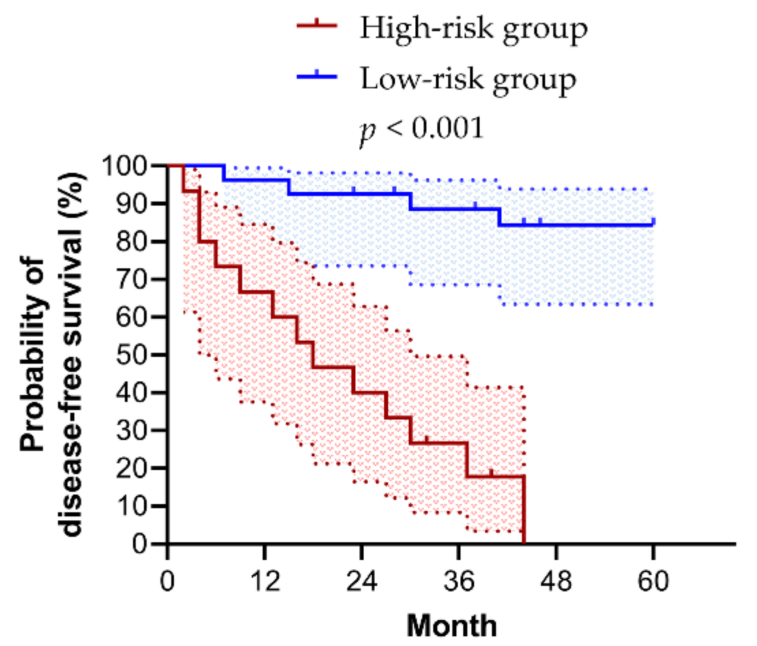

(b)

Figure 3. Kaplan-Meier curves of the predictive models of five-year prognoses. Comparison of (a) five-year local recurrence rates and (b) five-year disease-free survival rates between high-risk and low-risk groups using log-rank tests. 
Table 6. Spearman's correlations between clinical characteristics, laryngeal EBV-related biomarkers, and laryngeal histological characteristics in patients with LSCC.

\begin{tabular}{|c|c|c|c|c|c|c|c|c|c|c|c|c|c|c|c|}
\hline Characteristics & $\begin{array}{l}\text { Male } \\
\text { Sex }\end{array}$ & $\begin{array}{c}\text { Age } \leq 63 \\
\text { years }\end{array}$ & $\begin{array}{l}\text { Cigarette } \\
\text { Smoking }\end{array}$ & $\begin{array}{c}\text { Alcohol } \\
\text { Consumption }\end{array}$ & $\begin{array}{c}\text { T-Stage } \\
\leq 2\end{array}$ & $\begin{array}{l}\mathrm{N}-\text { Stage } \\
=0\end{array}$ & $\underset{2}{\text { Stage }} \leq$ & $\begin{array}{l}\text { Laryngeal } \\
\text { EBV DNA } \\
\text { Positivity }\end{array}$ & $\begin{array}{c}E B E R \\
\text { Signal } \geq \\
0.04 \%\end{array}$ & $\begin{array}{c}B C L-2 \\
\text { Expression } \\
\leq 96.0 \%\end{array}$ & $\begin{array}{l}M Y C \text { Ex- } \\
\text { pression } \\
\leq \mathbf{0 . 5 0 \%}\end{array}$ & $\begin{array}{c}p 16 I N K 4 a \\
\text { Expression } \\
\leq 81.6 \%\end{array}$ & $\begin{array}{c}B 2 M \\
\text { Expression } \\
\leq 92.7 \% \\
\end{array}$ & $\begin{array}{c}C D 3 \\
\text { Expression } \\
\leq 4.9 \%\end{array}$ & $\begin{array}{c}C D 161 \\
\text { Expression } \\
\leq 69.9 \% \\
\end{array}$ \\
\hline Male sex & - & & & & & & & & & & & & & & \\
\hline Age $\leq 63$ years & -0.02 & - & & & & & & & & & & & & & \\
\hline Cigarette smoking & $0.54^{\mathrm{a}}$ & 0.08 & - & & & & & & & & & & & & \\
\hline $\begin{array}{l}\text { Alcohol } \\
\text { consumption }\end{array}$ & 0.27 & $0.31^{\mathrm{b}}$ & $0.37^{\mathrm{b}}$ & - & & & & & & & & & & & \\
\hline T-stage $\leq 2$ & 0.13 & -0.10 & 0.25 & -0.02 & - & & & & & & & & & & \\
\hline N-stage $=0$ & -0.07 & -0.01 & -0.06 & 0.07 & 0.20 & - & & & & & & & & & \\
\hline Stage $\leq 2$ & 0.02 & -0.15 & 0.10 & 0.02 & $0.44^{c}$ & $0.46^{c}$ & - & & & & & & & & \\
\hline $\begin{array}{l}\text { Laryngeal EBV DNA } \\
\text { positivity }\end{array}$ & $-0.34^{b}$ & -0.15 & -0.11 & 0.01 & -0.29 & -0.24 & -0.27 & - & & & & & & & \\
\hline$E B E R$ signal $\geq 0.04 \%$ & 0.03 & 0.24 & 0.18 & 0.14 & 0.06 & 0.14 & $0.31^{b}$ & -0.18 & - & & & & & & \\
\hline $\begin{array}{c}B C L-2 \text { expression } \leq \\
96.0 \%\end{array}$ & 0.06 & 0.20 & 0.15 & 0.20 & 0.07 & -0.18 & 0.22 & -0.09 & 0.20 & - & & & & & \\
\hline $\begin{array}{c}\text { MYC expression } \leq \\
0.50 \%\end{array}$ & -0.22 & -0.29 & 0.01 & -0.18 & 0.20 & 0.09 & 0.20 & 0.02 & 0.20 & 0.08 & - & & & & \\
\hline $\begin{array}{c}\text { p16INK4a expression } \\
\leq 81.6 \%\end{array}$ & 0.05 & $0.44^{\mathrm{c}}$ & 0.13 & 0.16 & 0.11 & 0.03 & 0.07 & -0.23 & 0.12 & $0.36^{\mathrm{b}}$ & 0.25 & - & & & \\
\hline $\begin{array}{c}B 2 M \text { expression } \leq \\
92.7 \%\end{array}$ & 0.03 & 0.01 & -0.07 & 0.12 & 0.06 & 0.02 & 0.05 & 0.20 & 0.15 & 0.13 & 0.12 & -0.21 & - & & \\
\hline $\begin{array}{c}C D 3 \text { expression } \leq \\
4.9 \%\end{array}$ & 0.12 & 0.09 & 0.10 & -0.03 & 0.11 & 0.08 & 0.18 & -0.20 & 0.09 & 0.30 & 0.09 & $0.32^{\mathrm{b}}$ & 0.19 & - & \\
\hline $\begin{array}{c}C D 161 \text { expression } \leq \\
69.9 \%\end{array}$ & 0.03 & 0.01 & -0.07 & 0.02 & -0.04 & 0.02 & 0.05 & 0.01 & 0.15 & $0.32^{b}$ & -0.20 & -0.30 & $0.42^{\mathrm{c}}$ & 0.19 & - \\
\hline
\end{tabular}

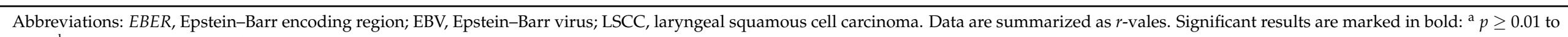
$<0.05,{ }^{\mathrm{b}} p \geq 0.001$ to $<0.01$, and ${ }^{\mathrm{c}} p<0.001$. 


\section{Discussion}

In this study, we found that patients with primary LSCC had a higher prevalence of EBV infection, compared to control patients. In addition to age $\geq 55$ years and cigarette smoking [37], EBV DNA positivity, BCL-2 expression $\geq 68.6 \%, B 2 \mathrm{M}$ expression $\geq 84.3 \%$, and CD161 expression $\geq 68.8 \%$ were independently associated with LSCC. Using this novel model, we accurately predicted the development of LSCC. Moreover, EBER signals $\geq 0.04 \%$ and $C D 3$ expression $\leq 4.9 \%$ independently predicted five-year LR and DFS rates with or without adjustment for treatment modality. Using this innovative two-factor model, we could also accurately predict the five-year LR and DFS rates of LSCC.

Chronic viral infection is a risk factor for many cancers, such as hepatitis B virus/hepatocellular carcinoma, human papillomavirus/cervical cancer, and EBV/NPC. Quantifying EBV-related biomarker levels in tumor specimens could improve our understanding of the etiology of some laryngeal cancers, and assist in patient selection for treatment and followup protocols [38]. However, quantification in FFPE tissue is difficult due to ambiguous uncertainties and the absence of reliable detection methods. Determining the interactions between EBV-related biomarkers and host factors is also challenging. For translation purposes, we developed and validated risk and prognostic models based on EBV-related biomarkers in this case-control study of Taiwanese patients with primary LSCC.

Similar to other studies $[8,9,11]$, we found that EBV infection was significantly more frequent in patients with primary LSCC than in those with NMLL. However, we found that most EBERs were detected in the TILs of LSCC. The absence of latent EBV infection in the tumor tissue of LSCC suggests that its multistep nature of development may include "hit-and-run" carcinogenesis in the laryngeal epithelium. Notably, EBV DNA positivity was positively correlated with $B C L-2$ expression, an anti-apoptotic protein in tumor cells. $W p$, a viral promoter located within BamHI $W$ repeats of the EBV genome, activates the gene encoding the viral BCL-2 homolog BHRF1, thereby increasing apoptosis resistance [39]. Moreover, oncogenic EBV latent membrane protein 1 directly upregulates $B C L-2$ in NPC [40], potentially also contributing to LSCC [11].

Furthermore, we found that $B C L-2$ overexpression in LSCC was common $(80 \%)$. $B C L-2$ interacts with $H s p 90 \beta$, and may be involved in the anti-apoptotic progression of LSCC [41]. BCL-2 overexpression appears to be associated with a complete response to induction chemotherapy [16] and worse prognoses due to tumoral radioresistance [42]. Although BCL-2 overexpression might not contribute to the prognostic significance of LSCC development [43], we found that both EBV DNA positivity and BCL-2 overexpression in the larynx were independently associated with a risk of LSCC. Our results also indicate that $B C L-2$ expression is not related to tumor aggressiveness and prognosis after a single surgical treatment [42].

Regarding therapeutic prognoses, we found that $E B E R$ signals $\geq 0.04 \%$ and CD3 expression $\leq 4.9 \%$ were important prognostic factors for five-year LR and DFS in patients with LSCC. In our literature review, only a few patients with LSCC had positive EBER signals in the larynx $[39,44]$. Although positive EBER signals in LSCC tissues are considered poorer predictors of head and neck squamous cell carcinoma (HNSCC) [39], a small LSCC sample size (two and six cases of positive and negative EBER signals, respectively) is difficult to draw conclusions from. Therefore, this study was the first to identify a high laryngeal EBER signal as a poorer prognostic factor in LSCC. Since most EBER signals presented the peritumor microenvironment in our patients, we presumed that $E B E R s$ could induce the initial transformation of epithelial cells [45], and trigger cancer-related inflammation via the RIG-I pathway to promote tumor development and growth [46]; rather than downregulate $\mathrm{B} 2 \mathrm{M}$ expression to evade $\mathrm{T}$ cell-mediated cytotoxic immune responses [47], as according to previous NPC studies. Moreover, EBV-infected TILs of residual laryngeal tissue following curative treatment may increase the five-year LR risk via BHRF1-mediated apoptosis resistance [48]. Accordingly, mechanistic studies are warranted to confirm that EBV involves the EBER-mediated process of LSCC recurrence. 
Circulating EBV DNA is a robust biomarker for EBV-associated NPC [49] and lymphoma [50]. EBV VCA IgA positivity indicates previous repeated EBV infections or frequent reactivation of latent EBV in B cells [51]. Patients with LSCC might have a higher positive rate of EBV VCA IgA than healthy controls [52]. Therefore, both circulating EBV DNA and VCA IgA could be potential biomarkers for EBV-positive LSCC. In this study, neither circulating EBV DNA positivity nor serum EBV VCA IgA positivity was associated with laryngeal EBV DNA positivity and EBER nPI. Furthermore, the low positive rates of these peripheral blood biomarkers may limit their application in patients with LSCC.

Laryngeal cancer usually develops over many years, and approximately $80 \%$ of patients with LSCC are 60 years or older at first discovery [53]. In this study, an age $\geq 55$ years was associated with $B 2 M$ expression $\geq 84.3 \%$, and both were independent risk factors for LSCC development. Recently, B2M was validated as a key reference gene in laryngeal and hypopharyngeal cancers, highlighting its suitability for investigating target gene expression [54]. Interestingly, its expression in patients with EBV-positive cancer was significantly higher than that in patients with EBV-negative cancer [55]. The $B 2 M$ protein forms the light chain of the class I major histocompatibility complex, and is important for antigen recognition by cytotoxic T cells [56]. Overexpression of $B 2 M$ is found in solid tumors and blood-borne malignancies, and is associated with advanced disease and poor prognosis [57]. Although B2M expression was not associated with LSCC stage and prognosis, it was an independent risk factor for LSCC development.

Furthermore, cigarette smoking is strongly associated with an increased risk of LSCC in a dose-response and time-response manner [58]. Although significant smoking trend reductions have led to the declined global burden of LSCC in developed countries, smoking continues to trend upward in low socioeconomic countries, potentially inducing an increased burden of LSCC in the future [59]. Notably, smoking prevention programs conducted in health care settings and interventions, including enhancement of interpersonal communication and support strategies, can be effective in preventing smoking behavior for three months to four years in children and adolescents [60]. However, the latest meta-analysis indicated that findings which suggest that reduction-to-quit is more effective than no treatments were inconclusive and of low certainty [61]. Nevertheless, the risk of developing LSCC can be reduced by smoking cessation for $\geq 15$ years [58].

CD161 is expressed on natural killer (NK) cells and subpopulations of T lymphocytes, such as invariant natural killer T cells (iNKT), $\mathrm{CD} 4^{+} \mathrm{CD} 161^{+} \mathrm{T}$ cells, and $\mathrm{CD} 8^{+} \mathrm{CD} 161^{+}$ $\mathrm{T}$ cells [62]. These cells may play significant roles in tumor development [63], and may regulate the immune response in the tumor microenvironment [64]. However, the effect of CD161 expression on tumor development remains unclear. Patients with LSCC had a significantly lower percentage of circulating iNKT cells, compared to healthy controls [65], whereas circulating $\mathrm{CD} 4^{+} \mathrm{CD} 161^{+} \mathrm{T}$ cells, representing a memory $\mathrm{T}$ cell population, significantly increased in cancer patients [66]. Notably, $\mathrm{CD}^{+} \mathrm{CD} 161^{+} \mathrm{T}$ cell-specific EBV infections might express high levels of anti-apoptotic molecules to survive hostile inflammatory conditions, also involving the pathogenesis of tumor tissues [67]. Furthermore, we found that CD161 overexpression in LSCC was not uncommon, and correlated with $B C L-2$ overexpression and low $p 16 I N K 4 a$ expression. However, both CD161 and BCL-2 overexpression were independent risk factors for LSCC development.

In patients with LSCC, low CD3 expression was associated with a poor prognosis. Similarly, a high infiltration of $\mathrm{CD}^{+} \mathrm{T}$ lymphocytes was associated with a significantly better prognosis in patients with HNSCC [68]. In patients with HNSCC, low CD3 mRNA levels have been found to be worse predictors of 8-year DFS [69]. Therefore, dysfunction of the immune response in removing the oncogenic EBV infection may increase the risk of cancer progression. Although we did not find a significant association between $C D 3$ expression and EBV infection in the larynx, patients with LSCC and EBV infection may have a lower proportion of late activated $\mathrm{T}$ lymphocytes in the peripheral blood compared to patients without EBV infection [70]. Therefore, investigating peripheral blood T lym- 
phocytes may help us to comprehensively understand the possible mechanisms of LR and relapse in EBV-related LSCC.

Our study had some limitations. First, when considering high EBV DNA positivity, false positivity is a real risk; PCR, which was used for EBV DNA detection, is extremely sensitive, and the EBER signal was minimal. However, we applied strict anticontamination measures in the laboratory to reduce the risk of contamination [27]. Second, in some patients, insufficient tissue and low-quality biomarkers limited the sample size; despite the use of TMAs to reduce the lesion volume required for comprehensive comparisons. Nevertheless, larger sample sizes may provide only modest benefits in complex statistical analyses.

\section{Conclusions}

Taken together, our study is the most comprehensive analysis to date of the clinical and histological manifestations, and etiology of primary LSCCs and five-year prognoses following definitive treatment. We identified EBV DNA positivity, older age, cigarette smoking, and higher expression of $B C L-2, B 2 M$, and CD161 as risk factors for primary LSCC. Furthermore, we identified high EBER signals and low $C D 3$ expression as independent predictors of five-year LR and DFS. Early prediction will enable the development of better treatments and follow-up strategies. Furthermore, as understanding the etiology of LSCC and reducing its LR rate is paramount, our data provide significant information that may assist in the accomplishment of these goals.

Author Contributions: Conceptualization: L.-A.L., T.-J.F., H.-Y.L., T.-C.C., C.-G.H., and T.-C.Y.; methodology: L.-A.L., K.-P.C., T.-C.C., and C.-G.H.; software: C.-J.K., C.-T.L., T.-C.C., and C.-G.H.; validation: L.-A.L., K.-P.C., T.-C.C., C.-G.H., and T.-C.Y.; formal analysis: L.-A.L., H.-H.C., and T.-C.Y.; investigation: L.-A.L., T.-J.F., H.-Y.L., C.-J.K., K.-P.C., and C.-T.L.; resources: L.-A.L., C.-G.H., and T.-C.Y.; data curation: C.-T.L. and T.-C.Y.; writing-original draft preparation: L.-A.L., T.-J.F., H.-H.C., K.-P.C., C.-G.H., and T.-C.Y.; writing-review and editing: H.-Y.L., C.-J.K., C.-T.L., and T.-C.C.; visualization: L.-A.L. and H.-H.C.; supervision: H.-Y.L., C.-T.L., T.-C.C., and T.-C.Y.; project administration: C.-J.K. and C.-G.H.; funding acquisition: L.-A.L. All authors have read and agreed to the published version of the manuscript.

Funding: This research was funded by the Chang Gung Medical Foundation, Taoyuan, Taiwan (grant numbers: 3C0661, 3C0662, 3C0663, and 3F1092). The APC was funded by the Chang Gung Medical Foundation, Taoyuan, Taiwan.

Institutional Review Board Statement: The study was conducted according to the guidelines of the Declaration of Helsinki and approved by the Institutional Review Board of the Chang Gung Medical Foundation, Taoyuan, Taiwan (protocol code 202100292B0 and date of approval 12 March 2021).

Informed Consent Statement: Patient consent was waived due to a retrospective medical review.

Data Availability Statement: The data presented in this study are available upon request from the corresponding author. The data are not publicly available due to ethical restrictions.

Acknowledgments: The authors would like to thank Ruo-Chi Wang and Chung-Fang Hsiao (Department of Otorhinolaryngology, Head and Neck Surgery, Linkou Chang Gung Memorial Hospital, Taoyuan City, Taiwan, R.O.C.) for their technical assistance.

Conflicts of Interest: The authors declare no conflict of interest.

\section{References}

1. Tai, P.; Yu, E.; Shiels, R.; Tonita, J. Long-term survival rates of laryngeal cancer patients treated by radiation and surgery, radiation alone, and surgery alone: Studied by lognormal and Kaplan-Meier survival methods. BMC Cancer 2005, 5, 13. [CrossRef] [PubMed]

2. Ferlay, J.; Soerjomataram, I.; Dikshit, R.; Eser, S.; Mathers, C.; Rebelo, M.; Parkin, D.M.; Forman, D.; Bray, F. Cancer incidence and mortality worldwide: Sources, methods and major patterns in GLOBOCAN 2012. Int. J. Cancer 2015, 136, E359-E386. [CrossRef] [PubMed]

3. Chang, J.; Fang, T.J.; Yung, K.; van Zante, A.; Miller, T.; Al-Jurf, S.; Schneider, S.; Courey, M. Clinical and histologic predictors of voice and disease outcome in patients with early glottic cancer. Laryngoscope 2012, 122, 2240-2247. [CrossRef] [PubMed] 
4. Bouvard, V.; Baan, R.; Straif, K.; Grosse, Y.; Secretan, B.; El Ghissassi, F.; Benbrahim-Tallaa, L.; Guha, N.; Freeman, C.; Galichet, L.; et al. A review of human carcinogens-Part B: Biological agents. Lancet Oncol. 2009, 10, 321-322. [CrossRef]

5. de Oliveira, D.E.; Bacchi, M.M.; Macarenco, R.S.; Tagliarini, J.V.; Cordeiro, R.C.; Bacchi, C.E. Human papillomavirus and EpsteinBarr virus infection, p53 expression, and cellular proliferation in laryngeal carcinoma. Am. J. Clin. Pathol. 2006, 126, $284-293$. [CrossRef]

6. Ndiaye, C.; Mena, M.; Alemany, L.; Arbyn, M.; Castellsagué, X.; Laporte, L.; Bosch, F.X.; de Sanjosé, S.; Trottier, H. HPV DNA, E6/E7 mRNA, and p16INK4a detection in head and neck cancers: A systematic review and meta-analysis. Lancet Oncol. 2014, 15, 1319-1331. [CrossRef]

7. Huang, C.G.; Lee, L.A.; Fang, T.J.; Li, H.Y.; Liao, C.T.; Kang, C.J.; Yen, T.C.; Tsao, K.C.; Chen, T.C.; Shih, S.R. Human papillomavirus infection is not associated with laryngeal squamous cell carcinoma in Taiwan. J. Microbiol. Immunol. Infect 2020, 53, 79-86. [CrossRef]

8. Jeng, K.C.; Hsu, C.Y.; Liu, M.T.; Chung, T.T.; Liu, S.T. Prevalence of Taiwan variant of Epstein-Barr virus in throat washings from patients with head and neck tumors in Taiwan. J. Clin. Microbiol. 1994, 32, 28-31. [CrossRef]

9. Zheng, Y.; Xia, P.; Zheng, H.C.; Takahashi, H.; Masuda, S.; Takano, Y. The screening of viral risk factors in tongue and pharyngolaryngeal squamous carcinoma. Anticancer. Res. 2010, 30, 1233-1238.

10. Brichacek, B.; Hirsch, I.; Sibl, O.; Vilikusova, E.; Vonka, V. Association of some supraglottic laryngeal carcinomas with EB virus. Int. J. Cancer 1983, 32, 193-197. [CrossRef]

11. Foltyn, S.; Strycharz-Dudziak, M.; Drop, B.; Boguszewska, A.; Polz-Dacewicz, M. Serum EBV antibodies and LMP-1 in Polish patients with oropharyngeal and laryngeal cancer. Infect Agent. Cancer 2017, 12, 31. [CrossRef]

12. Callaghan, D.J.; Conner, B.R.; Strauss, M. Epstein-Barr virus antibody titers in cancer of the head and neck. Arch. Otolaryngol. 1983, 109, 781-784. [CrossRef]

13. Yang, H.J.; Cho, Y.J.; Kim, H.S.; Chang, M.S.; Sung, M.W.; Kim, W.H. Association of p53 and BCL-2 expression with Epstein-Barr virus infection in the cancers of head and neck. Head Neck 2001, 23, 629-636. [CrossRef]

14. Muderris, T.; Rota, S.; Muderris, T.; Inal, E.; Fidan, I. Does Epstein-Barr virus infection have an influence on the development of laryngeal carcinoma? Detection of EBV by real-time polymerase chain reaction in tumour tissues of patients with laryngeal carcinoma. Braz. J. Otorhinolaryngol. 2013, 79, 418-423. [CrossRef]

15. Huang, S.C.M.; Tsao, S.W.; Tsang, C.M. Interplay of viral infection, host cell factors and tumor microenvironment in the pathogenesis of nasopharyngeal carcinoma. Cancers 2018, 10, 106. [CrossRef]

16. Trask, D.K.; Wolf, G.T.; Bradford, C.R.; Fisher, S.G.; Devaney, K.; Johnson, M.; Singleton, T.; Wicha, M. Expression of Bcl-2 family proteins in advanced laryngeal squamous cell carcinoma: Correlation with response to chemotherapy and organ preservation. Laryngoscope 2002, 112, 638-644. [CrossRef]

17. Li, J.; Huang, X.; Xie, X.; Wang, J.; Duan, M. Human telomerase reverse transcriptase regulates cyclin D1 and G1/S phase transition in laryngeal squamous carcinoma. Acta Otolaryngol. 2011, 131, 546-551. [CrossRef]

18. Feenstra, M.; Bakema, J.; Verdaasdonk, M.; Rozemuller, E.; van den Tweel, J.; Slootweg, P.; de Weger, R.; Tilanus, M. Detection of a putative HLA-A $* 31012$ processed (intronless) pseudogene in a laryngeal squamous cell carcinoma. Genes Chromosomes Cancer 2000, 27, 26-34. [CrossRef]

19. Lagresle, C.; Gardie, B.; Eyquem, S.; Fasseu, M.; Vieville, J.C.; Pla, M.; Sigaux, F.; Bories, J.C. Transgenic expression of the p16 ${ }^{\text {INK4a }}$ cyclin-dependent kinase inhibitor leads to enhanced apoptosis and differentiation arrest of CD4 ${ }^{-} \mathrm{CD} 8^{-}$immature thymocytes. $J$. Immunol. 2002, 168, 2325-2331. [CrossRef]

20. Kesselring, R.; Thiel, A.; Pries, R.; Wollenberg, B. The number of CD161 positive Th17 cells are decreased in head and neck cancer patients. Cell Immunol. 2011, 269, 74-77. [CrossRef]

21. McShane, L.M.; Altman, D.G.; Sauerbrei, W.; Taube, S.E.; Gion, M.; Clark, G.M.; Statistics Subcommittee of the NCI-EORTC Working Group on Cancer Diagnostics. Reporting recommendations for tumor marker prognostic studies. J. Clin. Oncol. 2005, 23, 9067-9072. [CrossRef]

22. Edge, S.B.; Compton, C.C. The American Joint Committee on Cancer: The 7th edition of the AJCC cancer staging manual and the future of TNM. Ann. Surg. Oncol. 2010, 17, 1471-1474. [CrossRef]

23. Fang, T.J.; Courey, M.S.; Liao, C.T.; Yen, T.C.; Li, H.Y. Frozen margin analysis as a prognosis predictor in early glottic cancer by laser cordectomy. Laryngoscope 2013, 123, 1490-1495. [CrossRef]

24. Hsin, L.J.; Fang, T.J.; Tsang, N.M.; Chin, S.C.; Yen, T.C.; Li, H.Y.; Liao, C.T.; Chen, I.H. Tumor volumetry as a prognostic factor in the management of T4a laryngeal cancer. Laryngoscope 2014, 124, 1134-1140. [CrossRef]

25. Lee, L.A.; Fang, T.J.; Li, H.Y.; Huang, C.G.; Chen, T.C.; Liao, C.T.; Kang, C.J.; Chang, K.P.; Yen, T.C. Low expression of pRB predicts disease relapse in early glottic cancer treated with transoral laser microsurgery. Laryngoscope 2018, 129, E220-E226. [CrossRef]

26. Fountzilas, E.; Kotoula, V.; Angouridakis, N.; Karasmanis, I.; Wirtz, R.M.; Eleftheraki, A.G.; Veltrup, E.; Markou, K.; Nikolaou, A.; Pectasides, D.; et al. Identification and validation of a multigene predictor of recurrence in primary laryngeal cancer. PLoS ONE 2013, 8, e70429. [CrossRef]

27. Huang, C.G.; Lee, L.A.; Tsao, K.C.; Liao, C.T.; Yang, L.Y.; Kang, C.J.; Chang, K.P.; Huang, S.F.; Chen, I.H.; Yang, S.L.; et al. Human papillomavirus 16/18 E7 viral loads predict distant metastasis in oral cavity squamous cell carcinoma. J. Clin. Virol. 2014, 61, 230-236. [CrossRef] 
28. Hsu, C.L.; Chang, K.P.; Lin, C.Y.; Chang, H.K.; Wang, C.H.; Lin, T.L.; Liao, C.T.; Tsang, N.M.; Lee, L.Y.; Chan, S.C.; et al. Plasma Epstein-Barr virus DNA concentration and clearance rate as novel prognostic factors for metastatic nasopharyngeal carcinoma. Head Neck 2012, 34, 1064-1070. [CrossRef]

29. Lo, Y.M.; Chan, L.Y.; Lo, K.W.; Leung, S.F.; Zhang, J.; Chan, A.T.; Lee, J.C.; Hjelm, N.M.; Johnson, P.J.; Huang, D.P. Quantitative analysis of cell-free Epstein-Barr virus DNA in plasma of patients with nasopharyngeal carcinoma. Cancer Res. 1999, 59, 1188-1191.

30. Hsu, C.L.; Chan, S.C.; Chang, K.P.; Lin, T.L.; Lin, C.Y.; Hsieh, C.H.; Huang, S.F.; Tsang, N.M.; Lee, L.Y.; Ng, S.H.; et al. Clinical scenario of EBV DNA follow-up in patients of treated localized nasopharyngeal carcinoma. Oral Oncol. 2013, 49, 620-625. [CrossRef]

31. Chang, K.P.; Hsu, C.L.; Chang, Y.L.; Tsang, N.M.; Chen, C.K.; Lee, T.J.; Tsao, K.C.; Huang, C.G.; Chang, Y.S.; Yu, J.S.; et al. Complementary serum test of antibodies to Epstein-Barr virus nuclear antigen-1 and early antigen: A possible alternative for primary screening of nasopharyngeal carcinoma. Oral Oncol. 2008, 44, 784-792. [CrossRef] [PubMed]

32. Cho, Y.J.; Chang, M.S.; Park, S.H.; Kim, H.S.; Kim, W.H. In situ hybridization of Epstein-Barr virus in tumor cells and tumorinfiltrating lymphocytes of the gastrointestinal tract. Hum. Pathol. 2001, 32, 297-301. [CrossRef] [PubMed]

33. Braun, M.; Kirsten, R.; Rupp, N.J.; Moch, H.; Fend, F.; Wernert, N.; Kristiansen, G.; Perner, S. Quantification of protein expression in cells and cellular subcompartments on immunohistochemical sections using a computer supported image analysis system. Histol. Histopathol. 2013, 28, 605-610. [CrossRef] [PubMed]

34. Youden, W.J. Index for rating diagnostic tests. Cancer 1950, 3, 32-35. [CrossRef]

35. Heagerty, P.J.; Lumley, T.; Pepe, M.S. Time-dependent ROC curves for censored survival data and a diagnostic marker. Biometrics 2000, 56, 337-344. [CrossRef] [PubMed]

36. Steyerberg, E.W.; Eijkemans, M.J.; Harrell, F.E., Jr.; Habbema, J.D. Prognostic modeling with logistic regression analysis: In search of a sensible strategy in small data sets. Med. Decis. Mak. 2001, 21, 45-56. [CrossRef]

37. Maier, H.; Gewelke, U.; Dietz, A.; Heller, W.D. Risk factors of cancer of the larynx: Results of the Heidelberg case-control study. Otolaryngol. Head Neck Surg. 1992, 107, 577-582. [CrossRef]

38. Turunen, A.; Rautava, J.; Grenman, R.; Syrjanen, K.; Syrjanen, S. Epstein-Barr virus (EBV)-encoded small RNAs (EBERs) associated with poor prognosis of head and neck carcinomas. Oncotarget 2017, 8, 27328-27338. [CrossRef]

39. Kelly, G.L.; Long, H.M.; Stylianou, J.; Thomas, W.A.; Leese, A.; Bell, A.I.; Bornkamm, G.W.; Mautner, J.; Rickinson, A.B.; Rowe, M. An Epstein-Barr virus anti-apoptotic protein constitutively expressed in transformed cells and implicated in burkitt lymphomagenesis: The Wp/BHRF1 link. PLoS Pathog. 2009, 5, e1000341. [CrossRef]

40. Young, L.S.; Rickinson, A.B. Epstein-Barr virus: 40 years on. Nat. Rev. Cancer 2004, 4, 757-768. [CrossRef]

41. Li, S.; Li, J.; Hu, T.; Zhang, C.; Lv, X.; He, S.; Yan, H.; Tan, Y.; Wen, M.; Lei, M.; et al. Bcl-2 overexpression contributes to laryngeal carcinoma cell survival by forming a complex with Hsp90ß. Oncol. Rep. 2017, 37, 849-856. [CrossRef]

42. Gioacchini, F.M.; Alicandri-Ciufelli, M.; Rubini, C.; Magliulo, G.; Re, M. Prognostic value of Bcl-2 expression in squamous cell carcinoma of the larynx: A systematic review. Int. J. Biol. Markers 2015, 30, e155-e160. [CrossRef]

43. Douglas, C.M.; Bernstein, J.M.; Ormston, V.E.; Hall, R.C.; Merve, A.; Swindell, R.; Valentine, H.R.; Slevin, N.J.; West, C.M.; Homer, J.J. Lack of prognostic effect of carbonic anhydrase-9, hypoxia inducible factor-1alpha and bcl-2 in 286 patients with early squamous cell carcinoma of the glottic larynx treated with radiotherapy. Clin. Oncol. 2013, 25, 59-65. [CrossRef]

44. McLemore, M.S.; Haigentz, M., Jr.; Smith, R.V.; Nuovo, G.J.; Alos, L.; Cardesa, A.; Brandwein-Gensler, M. Head and neck squamous cell carcinomas in HIV-positive patients: A preliminary investigation of viral associations. Head Neck Pathol. 2010, 4, 97-105. [CrossRef]

45. Yoshizaki, T.; Endo, K.; Ren, Q.; Wakisaka, N.; Murono, S.; Kondo, S.; Sato, H.; Furukawa, M. Oncogenic role of Epstein-Barr virus-encoded small RNAs (EBERs) in nasopharyngeal carcinoma. Auris Nasus Larynx 2007, 34, 73-78. [CrossRef]

46. Duan, Y.; Li, Z.; Cheng, S.; Chen, Y.; Zhang, L.; He, J.; Liao, Q.; Yang, L.; Gong, Z.; Sun, L.Q. Nasopharyngeal carcinoma progression is mediated by EBER-triggered inflammation via the RIG-I pathway. Cancer Lett. 2015, 361, 67-74. [CrossRef]

47. Kouvidou, C.; Rontogianni, D.; Tzardi, M.; Datseris, G.; Panayiotides, I.; Darivianaki, K.; Karidi, E.; Delides, G.; Kanavaros, P. Beta 2-microglobulin and HLA-DR expression in relation to the presence of Epstein-Barr virus in nasopharyngeal carcinomas. Pathobiology 1995, 63, 320-327. [CrossRef]

48. Fitzsimmons, L.; Cartlidge, R.; Chang, C.; Sejic, N.; Galbraith, L.C.A.; Suraweera, C.D.; Croom-Carter, D.; Dewson, G.; Tierney, R.J.; Bell, A.I.; et al. EBV BCL-2 homologue BHRF1 drives chemoresistance and lymphomagenesis by inhibiting multiple cellular pro-apoptotic proteins. Cell Death Differ. 2020, 27, 1554-1568. [CrossRef]

49. Lam, W.K.J.; Chan, K.C.A.; Lo, Y.M.D. Plasma Epstein-Barr virus DNA as an archetypal circulating tumour DNA marker. J. Pathol. 2019, 247, 641-649. [CrossRef]

50. Yalcin, B.; Kutluk, T.; Agbaba, S.K.; Demir, C.; Talim, B. Circulating Epstein-Barr virus DNA and cell-free DNA in pediatric lymphomas. Turk. J. Pediatr. 2020, 62, 541-550. [CrossRef]

51. Lo, S.; Ho, W.K.; Wei, W.I. Outcome of patients with positive Epstein-Barr virus serologic status in the absence of nasopharyngeal carcinoma in Hong Kong. Arch. Otolaryngol. Head Neck Surg. 2004, 130, 770-772. [CrossRef]

52. Morshed, K.; Polz-Dacewicz, M.; Szymanski, M.; Rajtar, B.; Ziaja-Soltys, M.; Golabek, W. Epstein-Barr virus antibodies in patients with laryngeal and hypopharyngeal cancer. Ann. Univ. Mariae Curie Sklodowska Med. 2003, 58, 227-231. 
53. Halmos, G.B.; Bras, L.; Siesling, S.; van der Laan, B.; Langendijk, J.A.; van Dijk, B.A.C. Age-specific incidence and treatment patterns of head and neck cancer in the Netherlands-A cohort study. Clin. Otolaryngol. 2018, 43, 317-324. [CrossRef]

54. Yin, W.Z.; Yang, Q.W.; Niu, K.; Ren, M.; He, D.; Song, W.Z. Validation of reference genes for the normalization of RT-qPCR expression studies on human laryngeal cancer and hypopharyngeal cancer. Eur. Rev. Med. Pharmacol. Sci. 2019, 23, 4199-4209. [CrossRef]

55. Keane, C.; Tobin, J.; Gunawardana, J.; Francis, S.; Gifford, G.; Gabrielli, S.; Gill, A.; Stevenson, W.; Talaulikar, D.; Gould, C.; et al. The tumour microenvironment is immuno-tolerogenic and a principal determinant of patient outcome in EBV-positive diffuse large B-cell lymphoma. Eur. J. Haematol. 2019, 103, 200-207. [CrossRef]

56. Rammensee, H.G.; Robinson, P.J.; Crisanti, A.; Bevan, M.J. Restricted recognition of beta 2-microglobulin by cytotoxic T lymphocytes. Nature 1986, 319, 502-504. [CrossRef]

57. Liu, C.; Yang, Z.; Li, D.; Liu, Z.; Miao, X.; Yang, L.; Zou, Q.; Yuan, Y. Overexpression of B2M and loss of ALK7 expression are associated with invasion, metastasis, and poor-prognosis of the pancreatic ductal adenocarcinoma. Cancer Biomark. 2015, 15, 735-743. [CrossRef]

58. Zuo, J.J.; Tao, Z.Z.; Chen, C.; Hu, Z.W.; Xu, Y.X.; Zheng, A.Y.; Guo, Y. Characteristics of cigarette smoking without alcohol consumption and laryngeal cancer: Overall and time-risk relation. A meta-analysis of observational studies. Eur. Arch. Otorhinolaryngol. 2017, 274, 1617-1631. [CrossRef]

59. Ramsey, T.; Guo, E.; Svider, P.F.; Lin, H.; Syeda, S.; Raza, S.N.; Fribley, A.M. Laryngeal cancer: Global socioeconomic trends in disease burden and smoking habits. Laryngoscope 2018, 128, 2039-2053. [CrossRef]

60. Duncan, L.R.; Pearson, E.S.; Maddison, R. Smoking prevention in children and adolescents: A systematic review of individualized interventions. Patient Educ. Couns. 2018, 101, 375-388. [CrossRef]

61. Lindson, N.; Klemperer, E.; Hong, B.; Ordonez-Mena, J.M.; Aveyard, P. Smoking reduction interventions for smoking cessation. Cochrane Database Syst. Rev. 2019, 9, CD013183. [CrossRef] [PubMed]

62. Konduri, V.; Oyewole-Said, D.; Vazquez-Perez, J.; Weldon, S.A.; Halpert, M.M.; Levitt, J.M.; Decker, W.K. CD8(+)CD161(+) T-Cells: Cytotoxic me0mory cells with high therapeutic potential. Front. Immunol. 2020, 11, 613204. [CrossRef] [PubMed]

63. Ostroumov, D.; Fekete-Drimusz, N.; Saborowski, M.; Kuhnel, F.; Woller, N. CD4 and CD8 T lymphocyte interplay in controlling tumor growth. Cell Mol. Life Sci. 2018, 75, 689-713. [CrossRef] [PubMed]

64. Iwahori, K. Cytotoxic CD8 ${ }^{+}$lymphocytes in the tumor microenvironment. Adv. Exp. Med. Biol. 2020, 1224, 53-62. [CrossRef] [PubMed]

65. Klatka, J.; Grywalska, E.; Hymos, A.; Krasowska, E.; Mielnik, M.; Siwicka-Gieroba, D.; Markowicz, J.; Trojanowski, P.; Olszanski, W.; Rolinski, J. Subpopulations of natural killer-T-like cells before and after surgical treatment of laryngeal cancer. Cent. Eur. J. Immunol. 2017, 42, 252-258. [CrossRef]

66. Iliopoulou, E.G.; Karamouzis, M.V.; Missitzis, I.; Ardavanis, A.; Sotiriadou, N.N.; Baxevanis, C.N.; Rigatos, G.; Papamichail, M.; Perez, S.A. Increased frequency of CD4+ cells expressing CD161 in cancer patients. Clin. Cancer Res. 2006, 12, 6901-6909. [CrossRef]

67. Turtle, C.J.; Swanson, H.M.; Fujii, N.; Estey, E.H.; Riddell, S.R. A distinct subset of self-renewing human memory CD8+ T cells survives cytotoxic chemotherapy. Immunity 2009, 31, 834-844. [CrossRef]

68. Schneider, K.; Marbaix, E.; Bouzin, C.; Hamoir, M.; Mahy, P.; Bol, V.; Gregoire, V. Immune cell infiltration in head and neck squamous cell carcinoma and patient outcome: A retrospective study. Acta Oncol. 2018, 57, 1165-1172. [CrossRef]

69. Lecerf, C.; Kamal, M.; Vacher, S.; Chemlali, W.; Schnitzler, A.; Morel, C.; Dubot, C.; Jeannot, E.; Meseure, D.; Klijanienko, J.; et al. Immune gene expression in head and neck squamous cell carcinoma patients. Eur. J. Cancer 2019, 121, 210-223. [CrossRef]

70. Klatka, J.; Hymos, A.; Szkatula-Lupina, A.; Grywalska, E.; Klatka, B.; Terpilowski, M.; Stepulak, A. T-lymphocyte activation is correlated with the presence of anti-EBV in patients with laryngeal squamous cell carcinoma. In Vivo 2019, 33, $2007-2012$. [CrossRef] 